\title{
Expression of cancer-associated fibroblast related proteins in metastatic breast cancer: an immunohistochemical analysis
}

\author{
Hye Min Kim, Woo Hee Jung and Ja Seung Koo*
}

\begin{abstract}
Background: Cancer-associated fibroblast (CAF) is the most studied element of the tumor microenvironment, although no relationship has been identified between expression of their related proteins and the metastasis site. The purpose of this study was to investigate the expression of CAF related proteins and their implications according to the metastasis site in metastatic breast cancer.

Methods: Immunohistochemical staining was used to evaluate the expression of CAF related proteins (podoplanin, prolyl 4-hydroxylase, FAP $\alpha$, S100A4, PDGFR $\alpha$, PDGFR $\beta$, and NG2) in tissue microarrays from 132 cases of metastatic breast cancer (bone metastasis: 32 cases, brain metastasis: 38 cases, liver metastasis: 10 cases, and lung metastasis: 52 cases). Breast cancer subtypes were classified as luminal A, luminal B, HER-2, and triple negative breast cancer, according to the immunohistochemical staining results for estrogen and progesterone receptors, HER-2, and Ki-67 and FISH results for HER-2. Tumors were classified as desmoplastic, sclerotic, normal-like, and inflammatory type, according to the histologic findings from the tumor stroma.

Results: Various CAF related protein expression profiles were observed, according to the metastasis site. For bone metastasis, the expression of stromal podoplanin, S100A4, and PDGFRa was significantly high. For lung metastasis, the expression of stromal PDGFR $\beta$ was significantly elevated $(p<0.001)$. For liver metastasis, significantly reduced expression of stromal S100A4 ( $p=0.002)$ and PDGFR $\alpha(p=0.011)$ was observed. Expression of CAF related proteins also differed according to the stromal phenotype. Desmoplastic stroma exhibited significantly elevated expression of stromal podoplanin ( $p<0.001)$, S100A4 ( $p<0.001)$, PDGFR $\alpha(p=0.010)$, and PDGFR $\beta(p=0.021)$. Inflammatory stroma exhibited significantly elevated expression of stromal FAP $\alpha=0.044)$ and significantly reduced stromal S100A4 expression $(p<0.001)$. Sclerotic stroma exhibited significantly elevated tumoral FAP $\alpha(p=0.005)$ expression. For lung metastasis, shorter overall survival was significantly related to tumoral podoplanin expression $(p=0.006)$, stromal podoplanin expression ( $p=0.018$ ), tumoral prolyl 4-hydroxylase negativity $(p=0.016)$, and tumoral PDGFR $\alpha$ expression $(p=0.001)$.
\end{abstract}

Conclusion: For metastatic breast cancer, significant differences were observed in the expression of CAF related proteins, according to the metastasis site and stromal histologic phenotype.

Keywords: Breast cancer, Cancer-associated fibroblast, Molecular subtype, Stroma

\section{Background}

Breast cancers are associated with high morbidity and mortality rates, as they are prone to distant metastasis.

*Correspondence: kjs1976@yuhs.ac

Department of Pathology, Severance Hospital, Yonsei University College of Medicine, 50 Yonsei-ro, Seodaemun-gu, Seoul 120-752, South Korea
The major metastasis sites for breast cancer are the lungs, brain, liver, and bone [1, 2], and most studies have been evaluated in brain and bone metastasis [3-8]. The most common mechanism for tumor metastasis is a reciprocal interaction between the tumor cell and host tissue, which is achieved by adhesion, proteolysis, invasion, and angiogenesis $[2,9]$. In addition, the importance of the 
tumor microenvironment in the process of metastasis has recently emerged. Among the various elements of the tumor microenvironment, cancer-associated fibroblast (CAF) is the most studied, and are currently considered most important element in this microenvironment [10]. Various proteins have been suggested as markers for CAF, including $\alpha$-SMA [11], tenascin-C [12], chondroitin sulfate proteoglycan (NG2) [13], platelet-derived growth factor receptors (PDGFR) $\alpha / \beta$ [14], fibroblast activation protein (FAP) [15], podoplanin [16], prolyl 4-hydroxylase [17], and fibroblast-specific protein (FSP)-1/S100A4 [13]. Therefore, it appears that CAF may consist of various functional subtypes. In one recent study, CAF were classified into various subsets, such as FAP $\alpha$ type, FSP1 type, PDGFR $\alpha$ type, and PDGFR $\beta$ type [18]. These subsets all showed different characteristics; this supports the hypothesis that CAF are comprised of diverse phenotypes.

To explain the unique metastasis pattern for each cancer, the "seed and soil" hypothesis has been proposed, which explains how a specific tumor (the seed) survives in a specific visceral organ (the soil) [19]. Similarly, different characteristic findings have been reported according to the site of metastasis for metastatic breast cancer. For example, previous studies have demonstrated that brain metastasis was related to young age, estrogen-receptor (ER) negativity, prior lung metastasis, HER-2 overexpression, epidermal growth factor receptor (EGFR) overexpression, and the basal subtype [5-7]. In addition, bone metastasis was related to a lower histologic grade, ER positivity, ER positivity and progesterone receptor (PR) negativity, strand growth patterns, and the presence of fibrotic foci in invasive ductal carcinoma $[4,20,21]$. Thus, as the characteristics of metastatic breast cancer differed according to the site of metastasis, it is likely that there are similar phenotypic differences for CAF, although few studies have evaluated this topic. Therefore, the purpose of this study was to investigate the expression of CAF related proteins and their implications, according to the metastasis site in metastatic breast cancer.

\section{Methods}

\section{Patient selection}

This study retrospectively reviewed cases of invasive primary breast cancer with metastasis to distant organs (liver, lungs, brain, and bone) from the records of the Department of Pathology, Severance Hospital, South Korea. Only patients that were diagnosed with invasive ductal carcinoma were included, which provided a total of 132 cases, including 49 cases with paired primary and metastasized tumors. All slides from these cases were reviewed, and the pathologic parameters were evaluated by 2 pathologists (JSK and WHJ). The histologic grade was assessed using the Nottingham grading system [22]. The study design was reviewed and approved by our Institutional Review Board.

For the invasive ductal carcinomas, the tumor stroma was classified according to the microscopic findings as desmoplastic type (stroma consisting of cellular fibroblast/myofibroblast proliferation), sclerotic type (stroma with fibrotic collagenous components and minimal cellular components), pauci-stromal type (minimal stromal tissue near the tumor), or inflammatory type (stroma consisting of inflammatory cells, such as lymphocytes).

\section{Tissue microarrays}

On H\&E-stained slides of tumors, a representative area was selected, and a corresponding spot was marked on the surface of a paraffin block. Using a biopsy needle, the selected area was extracted, and a $3-\mathrm{mm}$ tissue core was placed into a $6 \times 5$ array. Tissues from the invasive tumor were extracted, and more than two tissue cores were extracted to minimize extraction bias. Each tissue core was assigned a unique tissue microarray location number, which was linked to a database that contained the other clinicopathological data.

\section{Immunohistochemistry}

The antibodies and dilutions that were used for the immunohistochemistry are listed in Additional file 1: Table S1. All immunohistochemistry was performed using the formalin-fixed and paraffin-embedded tissue sections. Briefly, $5-\mu \mathrm{m}$ sections were obtained using a microtome, transferred onto adhesive slides, and dried at $62^{\circ} \mathrm{C}$ for $30 \mathrm{~min}$. After incubation with the primary antibodies, immunodetection was performed using biotinylated anti-mouse immunoglobulin, followed by peroxidase-labeled streptavidin using a labeled streptavidin biotin kit with $3,3^{\prime}$-diaminobenzidine chromogen as the substrate. The primary antibody incubation step was omitted in the negative control. Positive control tissue was used as per the manufacturer's recommendation. The slides were subsequently counterstained with Harris hematoxylin.

\section{Interpretation of immunohistochemical staining}

All immunohistochemical markers were assessed via light microscopy. A cut-off value of $\geq 1 \%$ positively stained nuclei was used to define ER and PR positivity [23]. HER-2 staining was analyzed according to the American Society of Clinical Oncology/College of American Pathologists guidelines, using the following categories: $0=$ no immunostaining; $1+=$ weak incomplete membranous staining in $<10 \%$ of the tumor cells; $2+=$ complete membranous staining, either uniform or weak, in $\geq 10 \%$ of the tumor cells; and $3+=$ uniform intense 
membranous staining in $\geq 30 \%$ of the tumor cells [24]. For our analysis, HER-2 immunostaining was considered positive when strong $(3+)$ membranous staining was observed, whereas 0 or $1+$ cases were considered negative. Cases with 2+ HER-2 expression were evaluated for HER-2 amplification via fluorescent in situ hybridization (FISH).

Immunohistochemical markers for podoplanin, prolyl 4-hydroxylase, FAP $\alpha$, S100A4, PDGFR $\alpha$, PDGFR $\beta$, and NG2 were assessed via light microscopy. The stained slides were evaluated semi-quantitatively, as reported previously [25]. In brief, tumor and stromal cell staining was assessed as $0=$ negative or weak immunostaining in $<1 \%$ of the tumor/stroma, $1=$ focal expression in $1-10 \%$ of the tumor/stroma, $2=$ positive in $11-50 \%$ of the tumor/stroma, or $3=$ positive in $51-100 \%$ of the tumor/ stroma. These evaluations were performed for the entire tumor area, and scores of 2-3 were defined as positive for our analysis.

\section{Tumor phenotype classification}

In this study, we classified the phenotypes of breast cancer according to the immunohistochemical results for ER, PR, HER-2, Ki-67, and FISH results for HER-2, as follows: luminal A type $=\mathrm{ER}$ and/or PR positive, HER-2 negative, and a Ki-67 labeling index (LI) of $<14 \%$; HER-2 negative luminal $\mathrm{B}$ type $=\mathrm{ER}$ and/or $\mathrm{PR}$ positive and Ki-67 LI $\geq 14 \%$; HER-2 positive luminal B type $=\mathrm{ER}$ and/ or PR positive, and HER-2 overexpressed and/or amplified; HER-2 overexpression type $=\mathrm{ER}$ and PR negative, and HER-2 overexpressed or/and amplified; and triple negative breast cancer $(\mathrm{TNBC})$ type $=$ negative for $\mathrm{ER}$, PR, and HER-2 [26].

\section{Statistical analysis}

Data were analyzed using SPSS for Windows (version 12.0, SPSS Inc., Chicago, IL, USA). Chi Square tests and Fisher's exact tests were used for categorical variables, respectively. When analyzing data with multiple comparisons, we used the Bonferroni multiple comparison procedure to generate a corrected $p$ value. Statistical significance was set at a p value of $<0.05$. Kaplan-Meier survival curves and log-rank statistics were used to evaluate the time to tumor recurrence and overall survival. Multivariate regression analysis was performed using the Cox proportional hazards model.

\section{Results}

\section{Characteristics of the breast cancer cases}

Among the 132 patients, 32 (24.2\%) had bone metastasis, 38 (28.8\%) had brain metastasis, 10 (7.6\%) had liver metastasis, and 52 (39.4\%) had lung metastasis. The proportion of patients with ER positivity and PR positivity was significantly elevated in bone and liver metastasis $(\mathrm{p}<0.001)$, and the proportion of patients with HER-2 positivity was significantly elevated in brain metastasis $(\mathrm{p}=0.047)$. A higher proportion of luminal A type was observed in bone and liver metastasis, and a higher proportion of TNBC type was observed in brain and lung metastasis $(\mathrm{p}<0.001)$ (Additional file 1: Table S2). The immunohistochemical staining results and clinicopathologic characteristics of patients with metastatic breast cancer were determined in the metastatic tumor.

\section{Clinicopathological features according to the stromal phenotype}

The stromal phenotype was analyzed, and 38 (28.8\%) patients was classified as having desmoplastic stroma, 9 (6.8\%) had inflammatory stroma, 45 (34.1\%) had paucistroma, and 40 (30.3\%) had sclerotic stroma. The clinicopathological characteristics were investigated according to the stromal phenotype and the only significant differences in stromal phenotype was according to the metastatic site $(\mathrm{p}<0.001)$. The proportion of inflammatory and sclerotic stroma was higher in lung metastasis, desmoplastic stroma was more common in bone metastasis, and pauci-stroma was more common in brain metastasis (Table 1).

\section{Expression of CAF related proteins according to the metastasis site}

Analysis of the expression of CAF related proteins in metastatic tumor according to the metastasis site revealed no expression of PDGFR $\beta$ and NG2 in the tumor cells, and no expression of prolyl 4-hydroxylase in the stromal component. However, significant differences were observed in the expression of tumoral podoplanin $(\mathrm{p}=0.008)$, stromal podoplanin $(\mathrm{p}=0.047)$, tumoral prolyl 4-hydroxylase $(\mathrm{p}=0.001)$, stromal S100A4 $(\mathrm{p}=0.002)$, stromal PDGFR $\alpha(\mathrm{p}=0.011)$, and stromal PDGFR $\beta$ ( $\mathrm{p}<0.001)$ according to the metastasis site. Stromal podoplanin, S100A4, and PDGFR $\alpha$ expression was elevated in bone metastasis, while tumoral podoplanin and stromal PDGFR $\beta$ expression was elevated in lung metastasis. In liver metastasis, tumoral prolyl 4-hydroxylase expression was elevated, while stromal S100A4 and PDGFR $\alpha$ expression was reduced. Expression of tumoral podoplanin, stromal podoplanin, tumoral prolyl 4-hydroxylase, stromal PDGFR $\alpha$, and stromal PDGFR $\beta$ was reduced in brain metastasis (Figures 1,2).

Analysis of the expression of CAF related proteins based on molecular subtype of each metastatic site revealed that stromal S100A4 $(\mathrm{p}=0.015)$ and tumoral $\operatorname{PDGFR} \alpha(\mathrm{p}=0.037)$ was associated with bone metastasis, tumoral prolyl 4-hydroxylase $(\mathrm{p}=0.003)$ and tumoral S100A4 ( $\mathrm{p}=0.034)$ with brain metastasis, stromal podoplanin $(\mathrm{p}=0.019)$ and tumoral S100A4 $(\mathrm{p}=0.019)$ with 
Table 1 Clinicopathological characteristics of the patients according to the breast cancer stromal histologic phenotype

\begin{tabular}{|c|c|c|c|c|c|c|}
\hline Parameter & $\begin{array}{l}\text { Total } \\
\mathrm{N}=132(\%)\end{array}$ & $\begin{array}{l}\text { Desmoplastic } \\
\text { type } n=38(\%)\end{array}$ & $\begin{array}{l}\text { Inflammatory } \\
\text { type } n=9(\%)\end{array}$ & $\begin{array}{l}\text { Pauci-stroma } \\
\text { type } n=45(\%)\end{array}$ & $\begin{array}{l}\text { Sclerotic type } \\
n=40(\%)\end{array}$ & $p$ value \\
\hline Age (years) & & & & & & 0.600 \\
\hline$\leq 50$ & $68(51.5)$ & $21(55.3)$ & $5(55.6)$ & $25(55.6)$ & $17(42.5)$ & \\
\hline$>50$ & $64(48.5)$ & $17(44.7)$ & $4(44.4)$ & $20(44.4)$ & $23(57.5)$ & \\
\hline ER & & & & & & 0.339 \\
\hline Negative & $63(47.7)$ & $20(52.6)$ & $6(66.7)$ & $22(48.9)$ & $15(37.5)$ & \\
\hline Positive & $69(52.3)$ & $18(47.4)$ & $3(33.3)$ & $23(51.1)$ & $25(62.5)$ & \\
\hline PR & & & & & & 0.060 \\
\hline Negative & $91(68.9)$ & $28(73.7)$ & $7(77.8)$ & $35(77.8)$ & $21(52.5)$ & \\
\hline Positive & $41(31.1)$ & $10(26.3)$ & $2(22.2)$ & $10(22.2)$ & $19(47.5)$ & \\
\hline HER-2 & & & & & & 0.370 \\
\hline Negative & $89(67.4)$ & $28(73.7)$ & $4(44.4)$ & $29(64.4)$ & $28(70.0)$ & \\
\hline Positive & $43(32.6)$ & $10(26.3)$ & $5(55.6)$ & $16(35.6)$ & $12(30.0)$ & \\
\hline Molecular subtypes & & & & & & 0.063 \\
\hline Luminal A & 47 (35.6) & $15(39.5)$ & $3(33.3)$ & $12(26.7)$ & $17(42.5)$ & \\
\hline Luminal B & $23(17.4)$ & $3(7.9)$ & $0(0.0)$ & $11(24.4)$ & $9(22.5)$ & \\
\hline HER-2 & $27(20.5)$ & $7(18.4)$ & $5(55.6)$ & $8(17.8)$ & $7(17.5)$ & \\
\hline TNBC & $35(26.5)$ & $13(34.2)$ & $1(11.1)$ & $14(31.1)$ & $7(17.5)$ & \\
\hline Ki-67 LI (\%) & & & & & & 0.139 \\
\hline$\leq 14$ & $91(68.9)$ & $31(81.6)$ & $6(66.7)$ & $26(57.8)$ & $28(70.0)$ & \\
\hline$>14$ & $41(31.1)$ & $7(18.4)$ & $3(33.3)$ & $19(42.2)$ & $12(30.0)$ & \\
\hline Metastasis site & & & & & & $<0.001$ \\
\hline Bone & $32(24.2)$ & $18(47.4)$ & $0(0.0)$ & 4 (8.9) & $10(25.0)$ & \\
\hline Brain & $38(28.8)$ & $3(7.9)$ & $2(22.2)$ & 25 (55.6) & $8(20.0)$ & \\
\hline Liver & $10(7.6)$ & $0(0.0)$ & $0(0.0)$ & $0(0.0)$ & $10(25.0)$ & \\
\hline Lung & $52(39.4)$ & $17(44.7)$ & $7(77.8)$ & $16(35.6)$ & $12(30.0)$ & \\
\hline Patients death & $44(33.3)$ & $15(39.5)$ & $3(33.3)$ & $14(31.1)$ & $12(30.0)$ & 0.815 \\
\hline
\end{tabular}

Italic value indicates statistically significant $(\mathrm{p}<0.05)$.

TNBC triple negative breast cancer.

liver metastasis, and tumoral PDGFR $\alpha(\mathrm{p}=0.034)$ and stromal PDGFR $\alpha(\mathrm{p}=0.042)$ with lung metastasis (Additional file 1: Table S3).

\section{Expression of CAF related proteins according to the stromal phenotype}

We also investigated the expression of CAF related proteins according to the stromal phenotype, and observed significant differences in the expression of stromal podoplanin ( $\mathrm{p}<0.001)$, tumoral FAP $\alpha(\mathrm{p}=0.005)$, stromal FAP $\alpha(\mathrm{p}=0.044)$, stromal S100A4 ( $<<0.001)$, stromal $\operatorname{PDGFR} \alpha(\mathrm{p}=0.010)$, and stromal PDGFR $\beta(\mathrm{p}=0.021)$. Desmoplastic stroma exhibited high expression of stromal podoplanin, S100A4, PDGFR $\alpha$, and PDGFR $\beta$, while inflammatory stroma exhibited high stromal FAP $\alpha$ and low tumoral FAP $\alpha$ and stromal S100A4 expression. Sclerotic stroma exhibited low stromal FAP $\alpha$ and high tumoral FAP $\alpha$ expression, while pauci-stroma exhibited low expression of stromal podoplanin, S100A4, PDGFR $\alpha$, and PDGFR $\beta$ (Figures 3, 4).

\section{Correlation of CAF related protein expression with primary} and metastatic breast cancer

Significant differences were observed in the expression of stromal podoplanin $(p=0.002)$ and tumoral prolyl 4-hydroxylase $(\mathrm{p}=0.039)$ in primary and metastatic breast cancer. They were positive in primary cancer, but negatively converted in metastasis site in 42.9 and $22.9 \%$ cases, respectively (Figure 5). Each metastatic sitespecific analysis did not show any significant findings; metastatic tumors other than lung metastasis were not included in the analysis, because the number is too small.

\section{Correlation between CAF related protein expression and the clinicopathological factors}

The relationship between CAF related protein expression and the clinicopathological factors were investigated (Figure 6). Tumoral PDGFR $\alpha$ expression was associated with ER and PR negativity $(\mathrm{p}<0.001$ and $\mathrm{p}=0.001$, respectively). The stromal phenotype was associated with stromal podoplanin $(\mathrm{p}<0.001)$ and S100A4 $(\mathrm{p}<0.001)$ 


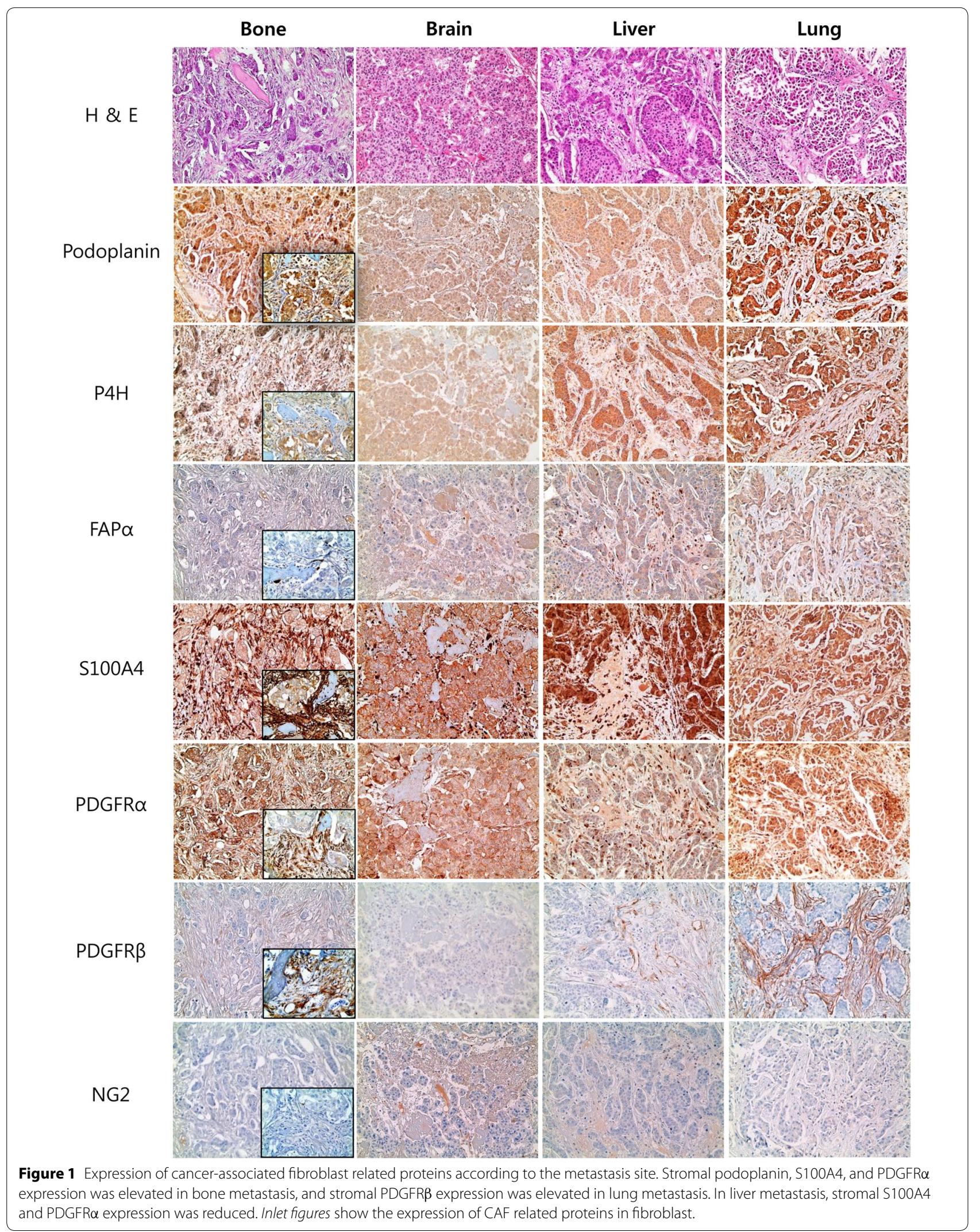




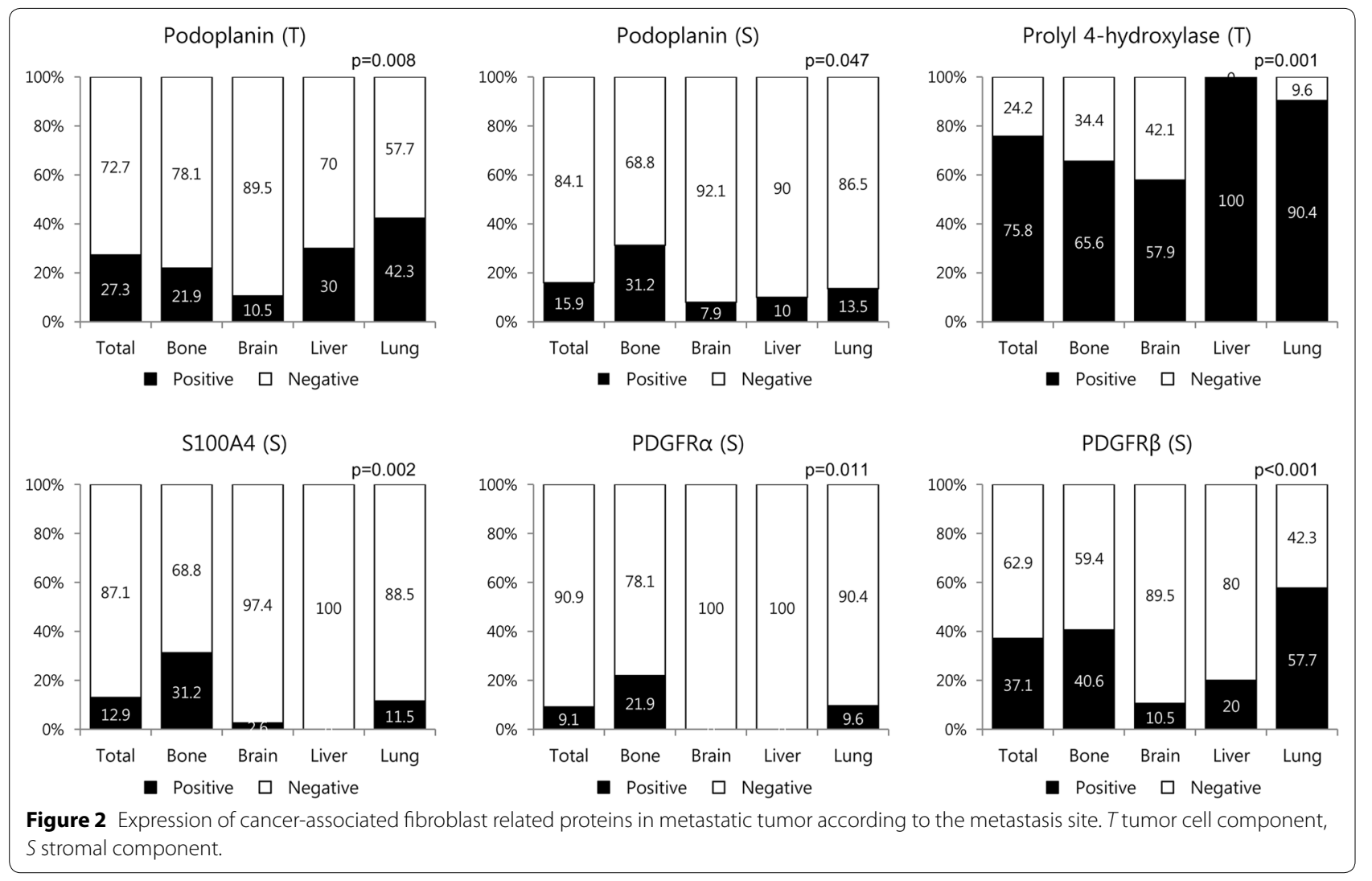

expression, with the desmoplastic stroma exhibiting high stromal podoplanin and S100A4 expression.

\section{Impact of CAF related protein expression on patient prognosis}

When the impact of CAF related protein expression on patient prognosis was analyzed, no factor was associated with shorter overall survival (OS) in the univariate analysis (Additional file 1: Table S4).

\section{Discussion}

Although no previous study has evaluated the expression of CAF related proteins according to the breast cancer metastasis site, the tumor stroma features are expected to be different, as different clinicopathological characteristics have been observed at different metastasis sites [27]. Therefore, we analyzed the expression of CAF related proteins according to the breast cancer metastasis site, and observed various different expression patterns. For example, the stromal expression of podoplanin, S100A4, and PDGFR $\alpha$ was high in bone metastasis, while PDGFR $\beta$ expression was high in lung metastasis. The high expression of podoplanin, S100A4, and PDGFR $\alpha$ in the CAF of bone metastasis is likely explained the characteristics of the bone environment.
In a previous study, different functional characteristics were identified according to the CAF subtype [18], with PDGFR $\alpha$ and S100A4 types of CAF being related to macrophage infiltration and macrophage recruitment $[28,29]$. It has also been suggested that bone is relevant to macrophage recruitment, because it is one of the main hematopoietic organs. In addition, a previous study has reported high expression of S100A4 in adipocyte-derived fibroblasts, which are a major desmoplastic stroma component in breast cancer [30]. As bone has numerous adipocytes in the marrow tissue, CAFs derived from marrow tissue are expected to exhibit high S100A4 expression. The second probable mechanism for our observed differences in CAF related protein expression is the differences in the metastatic cancer cell. For example, previous reports have reported metastasis sitespecific characteristics in metastatic breast cancer, with bone metastasis exhibiting a lower histologic grade, ER positivity, ER positivity and PR negativity, strand growth patterns, and the presence of fibrotic foci in invasive ductal carcinoma $[4,20,21]$. As the tumor stroma characteristics are formed via reciprocal interactions with cancer cells, various CAF phenotypes would also be expected if the cancer cell characteristics varied according to the site of metastasis. In addition, we observed 


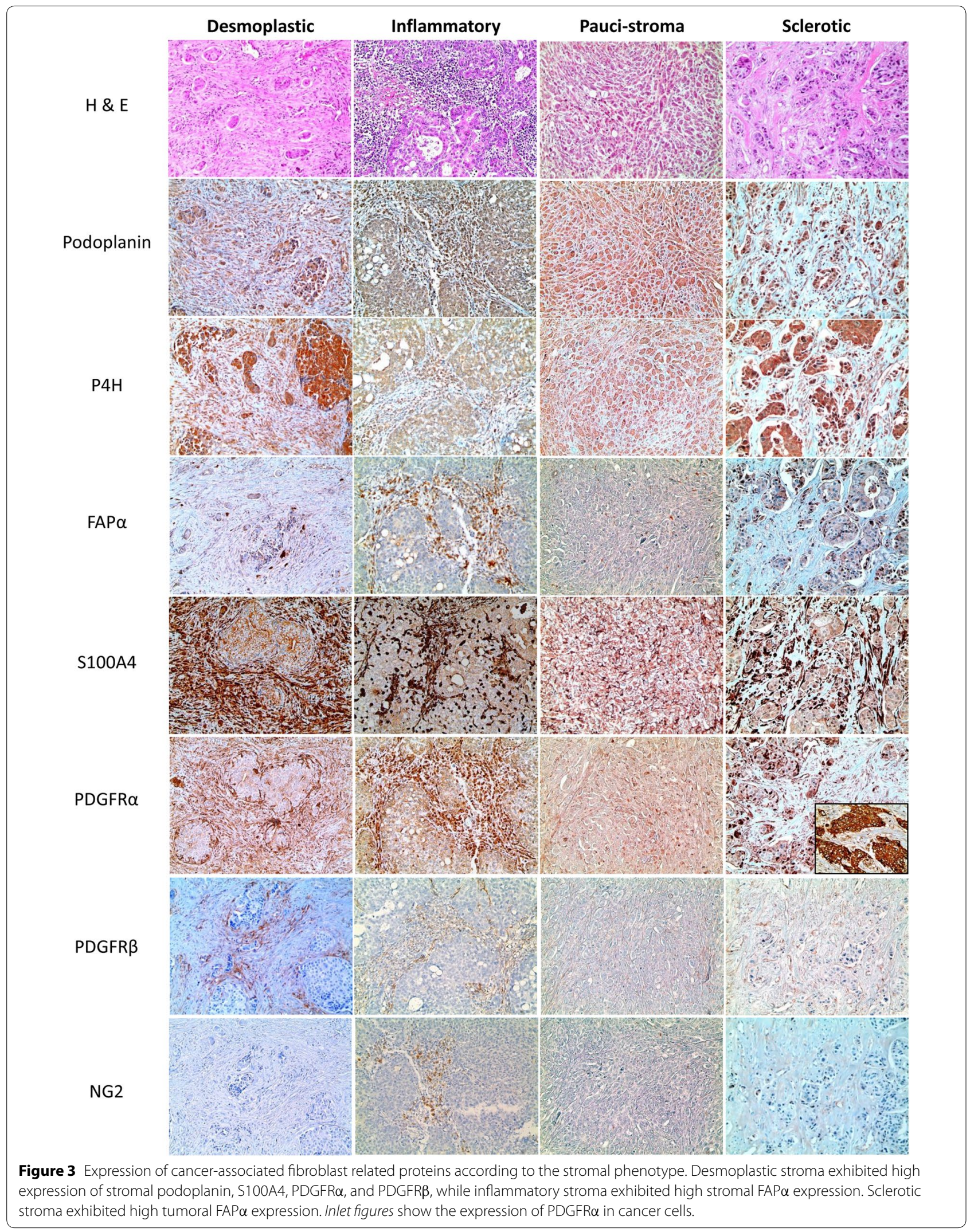



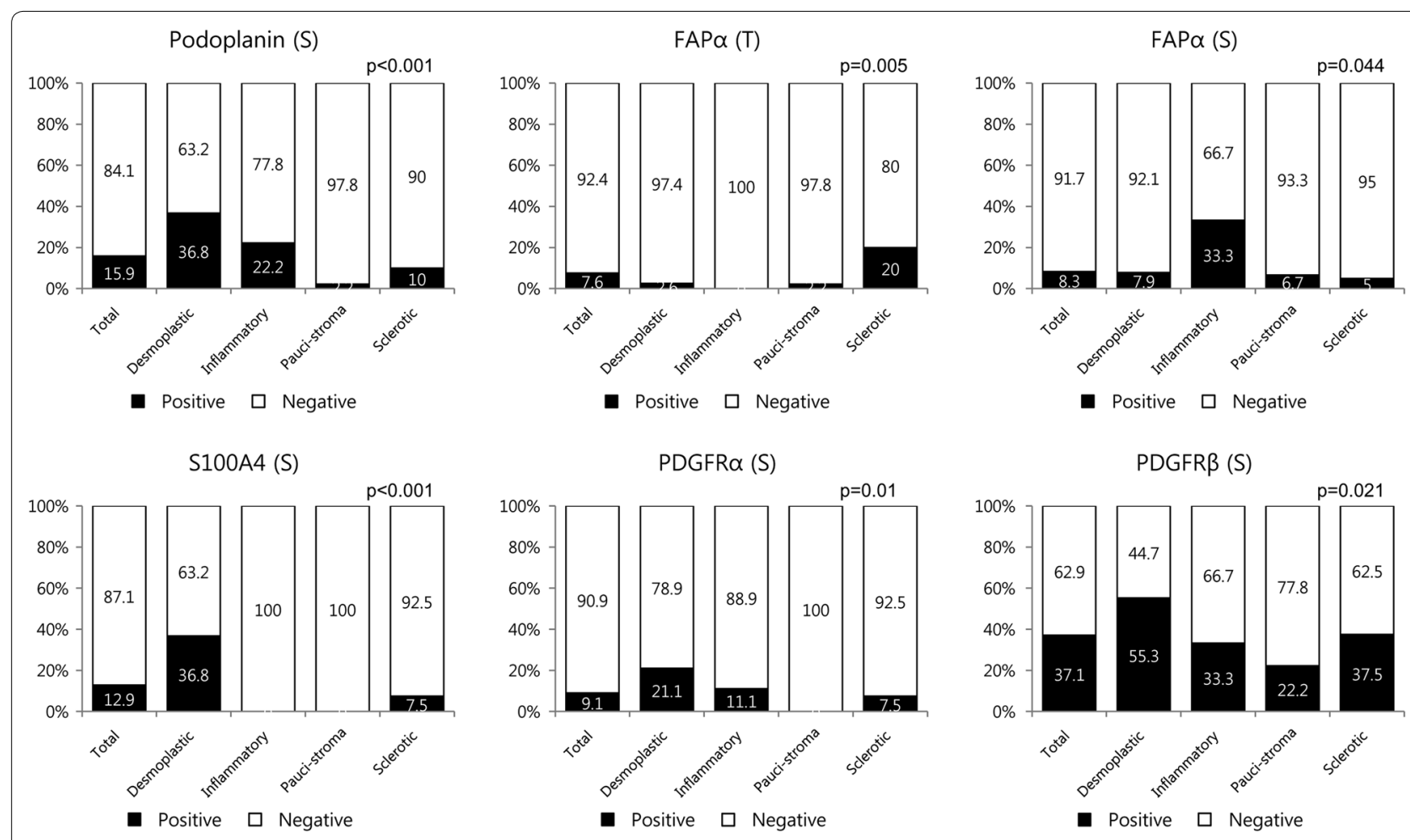

Figure 4 Expression of cancer-associated fibroblast related proteins in metastatic tumor according to the stromal phenotype. $T$ tumor cell component, $\mathbf{S}$ stromal component.

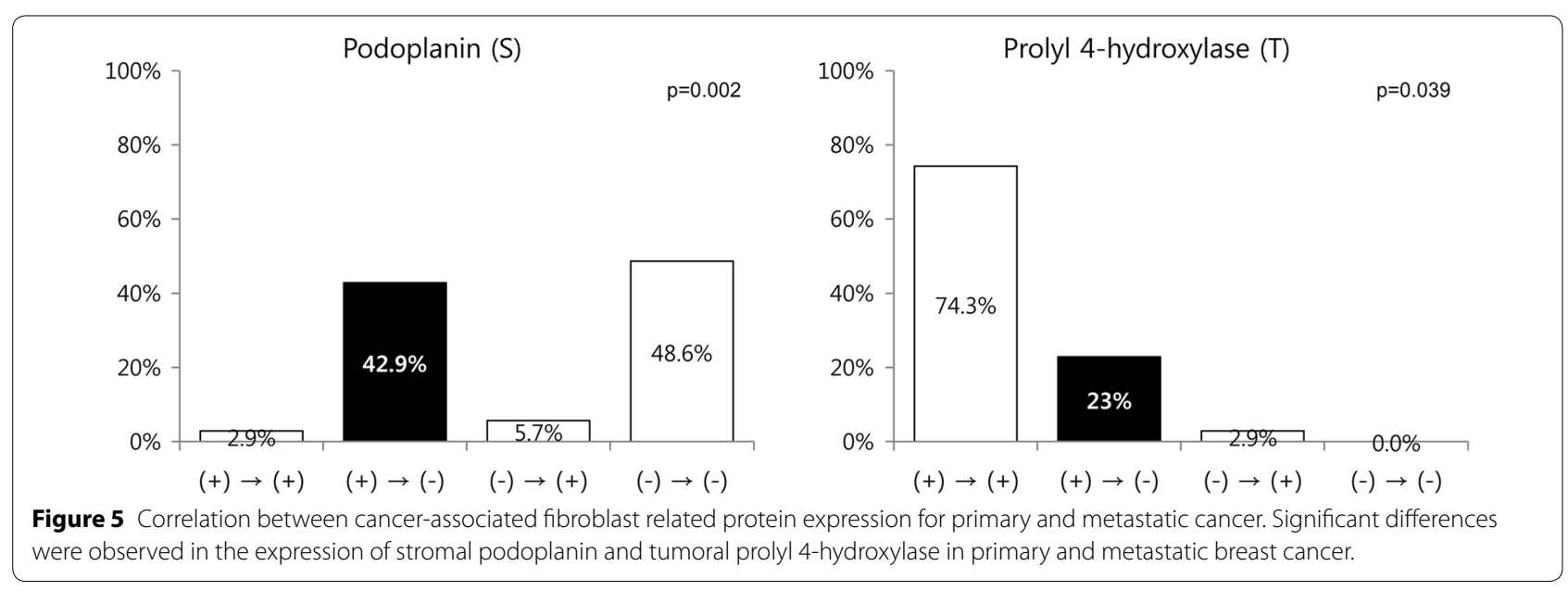

that the pathologic characteristics varied according to the metastasis site.

Therefore, different CAF profile might have influenced the tumor molecular subtype as well as different organ. However, our study indicates that CAF profile seems to be more related to different organ rather than tumor molecular subtype according to the following findings. First, there was no significant association between the expressions of CAF related proteins and molecular subtype by correlation analysis. Second, to evaluate the association between different organ and tumor molecular subtype in CAF profile, additional CAF profile by molecular subtype was evaluated according to metastatic site; if metastatic organ was identical, the items showing different expression of CAF related proteins by molecular subtype was fewer. 


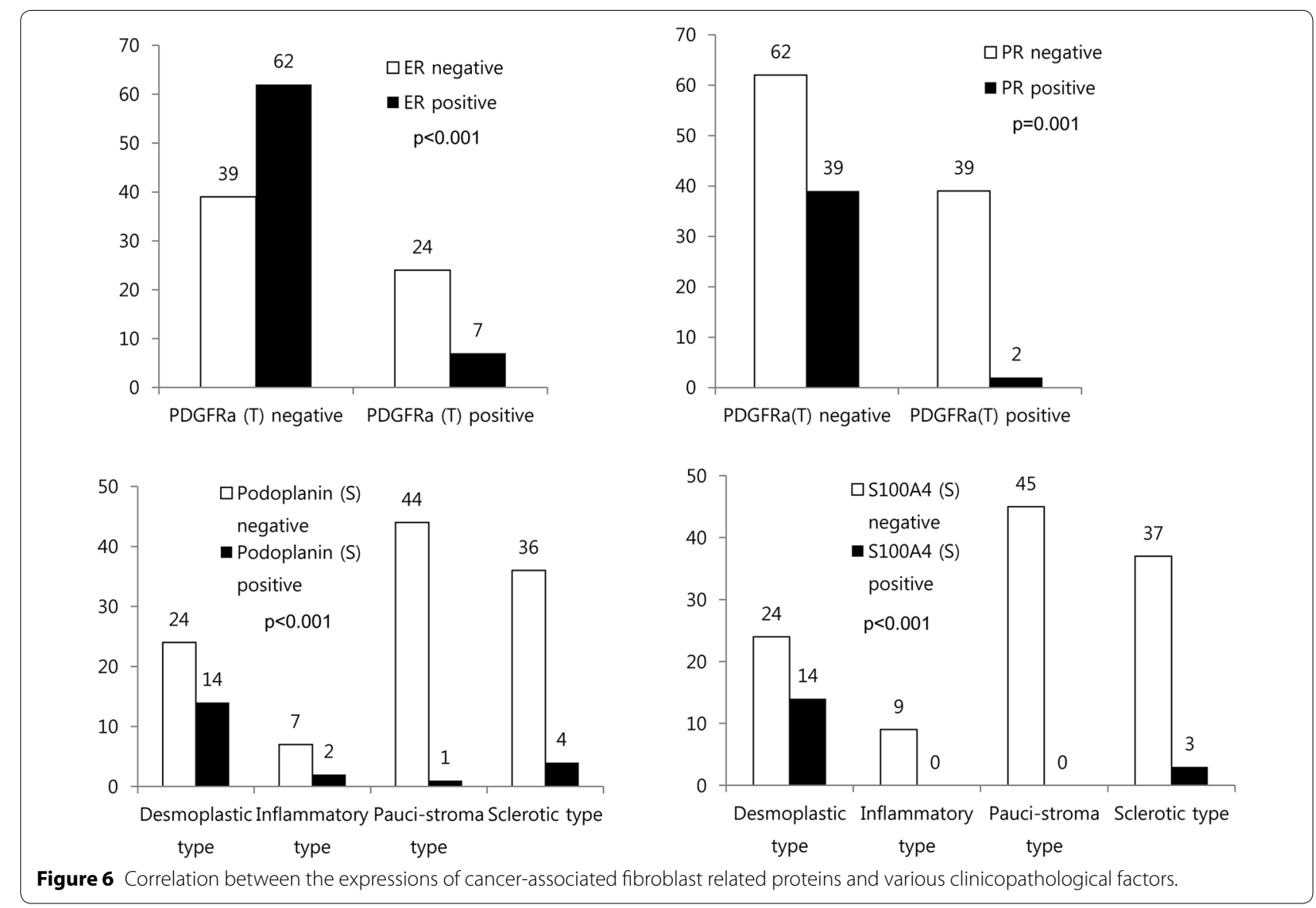

In lung metastasis, the CAF expression of PDGFR $\beta$ was high, and previous studies have reported a relationship between high interstitial fluid pressure and PDGFR $\beta$ type CAF [31]. Because the lungs have distinct histologic features, if a cancer cell metastasizes to a small interstitial tissue among the alveoli, it might encounter high interstitial fluid pressure, thereby promoting PDGFR $\beta$ expression. Furthermore, lung metastasis is known to be related to TNBC type; therefore, further investigation is needed to determine whether tumor cell characteristics might influence the CAF phenotype.

In this study, we demonstrated that CAF related protein expression varied according to the stromal histologic type, with desmoplastic stroma having high podoplanin, S100A4, PDGFR $\alpha$, and PDGFR $\beta$ expression, and inflammatory stroma having high FAP $\alpha$ expression. Although a previous study classified breast cancer tumor stroma according to their histologic findings [32], few study has compared the differences in CAF according to the histologic findings, therefore these results are not comparable. In addition, it has been noted that CAF markers have specific and unique features. For example, in breast cancer, the desmoplastic response appears to be mediated by
PDGF-AA signaling in PDGFR $\alpha$ type CAF [33]; this possibility is compatible with our findings. In addition, FAP $\alpha$ has been reported to possess an immunomodulatory function [18], which is consistent with our finding that inflammatory stroma has high FAP $\alpha$ expression. However, further investigation is needed to reveal the specific relationship between the tumor stroma histology and CAF characteristics.

The clinical implication of our findings is that CAF might be a potential anti-cancer therapeutic target. This target is particularly attractive, as it is genetically stable (relative to the cancer cell), exhibits distinct epigenetic changes within normal stromal cells, and can be targeted throughout the neoplasm process, as it supports and accompanies the cancer cell through the whole neoplasm spectrum [34]. Interestingly, several preclinical studies have targeted the CAF markers that we evaluated, and they reported that agents targeting CAF were effective in tumor inhibition [35-38]. However, to develop an effective cancer therapy, CAF-targeted treatments should exploit agents that target the specific CAF phenotype, and further studies are needed to determine which agents most effectively target each phenotype. 


\section{Conclusion}

In conclusion, the expression of CAF related proteins in stroma varies according to the breast cancer metastasis site and the stromal histologic phenotype.

\section{Additional file}

Additional file 1: Table S1. Source, clone, and dilution of the antibodies used. Table S2. Basal clinicopathologic characteristics of patients with metastatic breast cancer according to metastasis site. Table S3. Expression of CAF related proteins in the tumor cell compartment of metastatic breast cancer according to metastasis site.Table S4. Univariate analysis of the association between expression levels of CAF related proteins and overall survival by the log-rank test in metastatic breast cancer.

\section{Abbreviations}

CAF: cancer-associated fibroblast; NG2: chondroitin sulfate proteoglycan; PDGFR: platelet-derived growth factor receptors; FAP: fibroblast activation protein; FSP: fibroblast-specific protein; ER: estrogen-receptor; EGFR: epidermal growth factor receptor; PR: progesterone receptor; FISH: fluorescent in situ hybridization; TNBC: triple negative breast cancer; OS: overall survival.

\section{Authors' contributions}

HMK participated in the design of the study and performed the statistica analysis and carried out the immunoassays. WHJ participated in its design. JSK conceived the study, and participated in its design and coordination and helped to draft the manuscript. All authors read and approved the final manuscript.

\section{Acknowledgements}

This research was supported by Basic Science Research Program through the National Research Foundation of Korea (NRF) funded by the Ministry of Science, ICT and Future Planning (2015R1A1A1A05001209). This study was supported by a faculty research grant from Yonsei University College of Medicine for 2014 (6-2014-0131)

\section{Compliance with ethical guidelines}

\section{Competing interests}

The authors declare that they have no competing interests.

Received: 8 January 2015 Accepted: 29 June 2015

Published online: 11 July 2015

\section{References}

1. Weil RJ, Palmieri DC, Bronder JL, Stark AM, Steeg PS (2005) Breast cancer metastasis to the central nervous system. Am J Pathol 167:913-920

2. Woodhouse EC, Chuaqui RF, Liotta LA (1997) General mechanisms of metastasis. Cancer 80:1529-1537

3. Abali H, Celik I (2002) High incidence of central nervous system involvement in patients with breast cancer treated with epirubicin and docetaxel. Am J Clin Oncol 25:632-633

4. Colleoni M, O'Neill A, Goldhirsch A, Gelber RD, Bonetti M, Thurlimann B et al (2000) Identifying breast cancer patients at high risk for bone metastases. J Clin Oncol 18:3925-3935

5. Evans AJ, James JJ, Cornford EJ, Chan SY, Burrell HC, Pinder SE et al (2004) Brain metastases from breast cancer: identification of a high-risk group. Clin Oncol (R Coll Radiol) 16:345-349

6. Gaedcke J, Traub F, Milde S, Wilkens L, Stan A, Ostertag H et al (2007) Predominance of the basal type and HER-2/neu type in brain metastasis from breast cancer. Mod Pathol 20:864-870

7. Hicks DG, Short SM, Prescott NL, Tarr SM, Coleman KA, Yoder BJ et a (2006) Breast cancers with brain metastases are more likely to be estrogen receptor negative, express the basal cytokeratin CK5/6, and overexpress HER2 or EGFR. Am J Surg Pathol 30:1097-1104

8. Lorincz T, Toth J, Badalian G, Timar J, Szendroi M (2006) HER-2/neu genotype of breast cancer may change in bone metastasis. Pathol Oncol Res 12:149-152

9. Nicolson GL (1988) Organ specificity of tumor metastasis: role of preferential adhesion, invasion and growth of malignant cells at specific secondary sites. Cancer Metastasis Rev 7:143-188

10. Franco OE, Shaw AK, Strand DW, Hayward SW (2010) Cancer associated fibroblasts in cancer pathogenesis. Semin Cell Dev Biol 21:33-39

11. Desmouliere A, Guyot C, Gabbiani G (2004) The stroma reaction myofibroblast: a key player in the control of tumor cell behavior. Int J Dev Biol 48:509-517

12. De Wever O, Nguyen QD, Van Hoorde L, Bracke M, Bruyneel E, Gespach C et al (2004) Tenascin-C and SF/HGF produced by myofibroblasts in vitro provide convergent pro-invasive signals to human colon cancer cells through RhoA and Rac. FASEB J 18:1016-1018

13. Sugimoto $H$, Mundel TM, Kieran MW, Kalluri R (2006) Identification of fibroblast heterogeneity in the tumor microenvironment. Cancer Biol Ther 5:1640-1646

14. Pietras K, Sjoblom T, Rubin K, Heldin CH, Ostman A (2003) PDGF receptors as cancer drug targets. Cancer Cell 3:439-443

15. Kraman M, Bambrough PJ, Arnold JN, Roberts EW, Magiera L, Jones JO et al (2010) Suppression of antitumor immunity by stromal cells expressing fibroblast activation protein-alpha. Science 330:827-830

16. Kawase A, Ishii G, Nagai K, Ito T, Nagano T, Murata Y et al (2008) Podoplanin expression by cancer associated fibroblasts predicts poor prognosis of lung adenocarcinoma. Int J Cancer 123:1053-1059

17. Kojima Y, Acar A, Eaton EN, Mellody KT, Scheel C, Ben-Porath I et al (2010) Autocrine TGF-beta and stromal cell-derived factor-1 (SDF-1) signaling drives the evolution of tumor-promoting mammary stromal myofibroblasts. Proc Natl Acad Sci USA 107:20009-20014

18. Cortez E, Roswall P, Pietras K (2014) Functional subsets of mesenchymal cell types in the tumor microenvironment. Semin Cancer Biol 25:3-9

19. Paget $S$ (1889) The distribution of secondary growths in cancer of the breast. Lancet 1:571-572

20. Hasebe T, Imoto S, Yokose T, Ishii G, Iwasaki M, Wada N (2008) Histopathologic factors significantly associated with initial organ-specific metastasis by invasive ductal carcinoma of the breast: a prospective study. Hum Pathol 39:681-693

21. Wei B, Wang J, Bourne P, Yang Q, Hicks D, Bu H et al (2008) Bone metastasis is strongly associated with estrogen receptor-positive/progesterone receptor-negative breast carcinomas. Hum Pathol 39:1809-1815

22. Elston CW, Ellis IO (1991) Pathological prognostic factors in breast cancer. I. The value of histological grade in breast cancer: experience from a large study with long-term follow-up. Histopathology 19:403-410

23. Hammond ME, Hayes DF, Dowsett M, Allred DC, Hagerty KL, Badve S et al (2010) American Society of Clinical Oncology/College Of American Pathologists guideline recommendations for immunohistochemical testing of estrogen and progesterone receptors in breast cancer. J Clin Oncol 28:2784-2795

24. Wolff AC, Hammond ME, Schwartz JN, Hagerty KL, Allred DC, Cote RJ et al (2007) American Society of Clinical Oncology/College of American Pathologists guideline recommendations for human epidermal growth factor receptor 2 testing in breast cancer. J Clin Oncol 25:118-145

25. Henry LR, Lee HO, Lee JS, Klein-Szanto A, Watts P, Ross EA et al (2007) Clinical implications of fibroblast activation protein in patients with colon cancer. Clin Cancer Res 13:1736-1741

26. Goldhirsch A, Wood WC, Coates AS, Gelber RD, Thurlimann B, Senn HJ (2011) Strategies for subtypes-dealing with the diversity of breast cancer: highlights of the St. Gallen International Expert Consensus on the Primary Therapy of Early Breast Cancer 2011. Ann Oncol 22:1736-1747

27. Koo JS, Jung W, Jeong J (2010) Metastatic breast cancer shows different immunohistochemical phenotype according to metastatic site. Tumori 96:424-432

28. Zhang J, Chen L, Xiao M, Wang C, Qin Z (2011) FSP1 + fibroblasts promote skin carcinogenesis by maintaining MCP-1-mediated macrophage infiltration and chronic inflammation. Am J Pathol 178:382-390

29. Erez N, Truitt M, Olson P, Arron ST, Hanahan D (2010) Cancer-associated fibroblasts are activated in incipient neoplasia to orchestrate tumorpromoting inflammation in an NF-kappaB-dependent manner. Cancer Cell 17:135-147 
30. Bochet L, Lehuede C, Dauvillier S, Wang YY, Dirat B, Laurent V et al (2013) Adipocyte-derived fibroblasts promote tumor progression and contribute to the desmoplastic reaction in breast cancer. Cancer Res 73:5657-5668

31. Pietras K, Ostman A, Sjoquist M, Buchdunger E, Reed RK, Heldin CH et al (2001) Inhibition of platelet-derived growth factor receptors reduces interstitial hypertension and increases transcapillary transport in tumors. Cancer Res 61:2929-2934

32. Ahn S, Cho J, Sung J, Lee JE, Nam SJ, Kim KM et al (2012) The prognostic significance of tumor-associated stroma in invasive breast carcinoma. Tumour Biol 33:1573-1580

33. Shao ZM, Nguyen M, Barsky SH (2000) Human breast carcinoma desmoplasia is PDGF initiated. Oncogene 19:4337-4345

34. Ohlund D, Elyada E, Tuveson D (2014) Fibroblast heterogeneity in the cancer wound. J Exp Med 211:1503-1523
35. Haubeiss S, Schmid JO, Murdter TE, Sonnenberg M, Friedel G, van der Kuip H et al (2010) Dasatinib reverses cancer-associated fibroblasts (CAFs) from primary lung carcinomas to a phenotype comparable to that of normal fibroblasts. Mol Cancer 9:168

36. Brennen WN, Isaacs JT, Denmeade SR (2012) Rationale behind targeting fibroblast activation protein-expressing carcinoma-associated fibroblasts as a novel chemotherapeutic strategy. Mol Cancer Ther 11:257-266

37. Santos AM, Jung J, Aziz N, Kissil JL, Pure E (2009) Targeting fibroblast activation protein inhibits tumor stromagenesis and growth in mice. J Clin Invest 119:3613-3625

38. Scott AM, Wiseman G, Welt S, Adjei A, Lee FT, Hopkins W et al (2003) A Phase I dose-escalation study of sibrotuzumab in patients with advanced or metastatic fibroblast activation protein-positive cancer. Clin Cancer Res 9:1639-1647

\section{Submit your next manuscript to BioMed Central and take full advantage of:}

- Convenient online submission

- Thorough peer review

- No space constraints or color figure charges

- Immediate publication on acceptance

- Inclusion in PubMed, CAS, Scopus and Google Scholar

- Research which is freely available for redistribution

Submit your manuscript at

www.biomedcentral.com/submit 